\title{
CIUDADES INTELIGENTES, MARCO REGULATORIO EN COLOMBIA
}

\author{
Smart cities, regulatory framework in Colombia \\ Paola Astrid Ortega Sanchez¹, Hugo Felipe Salazar², Herman Antonio Fernández González \\ ${ }^{1}$ Escuela de Contaduría Pública, Universidad Pedagógica y Tecnológica de Colombia \\ (Sogamoso-Boyacá, Colombia).E-mail: paola.ortega01@uptc.edu.co \\ ${ }^{2}$ Escuela de Ingeniería Industrial, Universidad Pedagógica y Tecnológica de Colombia \\ (Sogamoso-Boyacá, Colombia).E-mail: felipe.salazar@uptc.edu.co \\ ${ }^{3}$ Escuela de Ingeniería Electrónica, Universidad Pedagógica y Tecnológica de Colombia \\ (Sogamoso-Boyacá, Colombia).E-mail: herman.fernandez@uptc.edu.co
}

(Recibido Abril 01 de 2018 y aceptado Agosto 21 de 2019)

\section{Resumen}

En este trabajo se presenta una revisión de literatura de los aspectos regulatorios y normativos que se han venido desarrollando bajo el concepto de ciudades inteligentes, smart Cities, en Colombia. A través del Ministerio de Tecnologías de la Información y las Comunicaciones (MinTIC) se ha propuesto elaborar una política pública con el objeto de estandarizar la implementación de tecnología en los diversos sectores de la sociedad colombiana durante los próximos cinco años. En este sentido, se han sintetizado los aspectos a regular de acuerdo con los siete componentes propuestos por MinTIC: competitividad, convivencia, cultura, hábitat, infraestructura, recursos y servicios, haciendo una breve descripción del marco normativo que podría aplicarse a cada uno de los componentes en particular.

Palabras clave: ciudades inteligentes, sostenibilidad, TIC

\begin{abstract}
This paper presents a literature review of the regulatory and normative aspects that have been developed under the concept of smart cities in Colombia. Through the Ministry of Information and Communication Technologies (MinTIC, Ministerio de Tecnologías de la Información y las Comunicaciones) has been proposed to develop a public policy in order to standardize the implementation of technology in several sectors of Colombian society over the next five years. In this sense, it has been synthesized the aspects to regulate in accordance with the seven components proposed by MinTIC: competitiveness, coexistence, culture, habitat, infrastructure, resources and services, making a brief description of the regulatory framework that could apply to each of these components.
\end{abstract}

Key words: smart cities, suistainability, ICT.

\section{INTRODUCCIÓN}

Se prevé que en el 2050 el $70 \%$ de la población mundial vivirá en ciudades, la urbanización sostenible se ha convertido en una prioridad política para las administraciones de todo el mundo [1]. Desde hace algunos años las Tecnologías de la Información y las
Comunicaciones (TIC) han venido desarrollando aplicaciones tecnológicas buscando promover el bienestar social. A través del Ministerio de Tecnologías de la Información y las Comunicaciones en Colombia (MinTIC) se han propuesto políticas que facilitan la inmersión a dichas tecnologías como es el caso de la construcción de ciudades inteligentes, smart cities. En el 2016 el MinTIC y la Cámara 
Colombiana de Informática y Telecomunicaciones (CCIT) organizaron el Foro Ciudades i $360^{\circ}$ dentro del contexto de "Colombia en el camino de las ciudades inteligentes" en el cual se abordaron temas como la vinculación del ciudadano en el uso de los medios electrónicos, la tecnología como eje transversal del progreso social de las ciudades y la innovación pública mediante las TIC [2].

El MinTIC cuenta con una plataforma interactiva con la que busca elaborar un documento que sirva como política pública para la implementación de la tecnología en los diversos sectores de la sociedad colombiana durante los próximos cinco años. Política encaminada a transformar las ciudades de Colombia en ciudades inteligentes, teniendo en cuenta las experiencias reales de la población en Colombia [3].

La Organización Internacional de Normalización (ISO, International Organization for Standardization), generó la norma ISO 37120:2014, la cual define y establece metodologías para un conjunto de indicadores con el objeto de orientar y medir el desempeño de los servicios de la ciudad y la calidad de vida [4]. De igual manera, se han creado comités con el objeto de facilitar la implantación de infraestructuras tecnológicas que permitan desarrollar un nuevo modelo de gestión de servicios urbanos basados en la eficiencia y la sostenibilidad. A manera de ejemplo, en Europa se ha creado el Foro Sectorial sobre Ciudades y Comunidades Inteligentes y Sostenibles (SF-SSCC, Sector Forum on Smart and Sustainable Cities and Communities), enero de 2017 [5]. Asimismo, en España el Comité Técnico de Normalización de AENOR AEN/CNT 178 "Ciudades Inteligentes", [6].

En este trabajo se presenta una revisión de los aspectos regulatorios y normativos que posiblemente permitirán establecer los estándares necesarios para la implementación de los diferentes elementos que conforman una ciudad inteligente. En la sección 2, se lleva a cabo una descripción del marco normativo que podría aplicarse a cada uno de los componentes que estructuran la inmersión al concepto de ciudades inteligentes en Colombia.
En la sección 3, se ilustra brevemente algunas de las normas que se han venido proponiendo internacionalmente por la ISO. Por último, se describe las conclusiones de este trabajo.

\section{REGULACIÓN NORMATIVA}

La constitución política de 1991, como referente constitucional desarrolla en su art 15 el derecho fundamental a la intimidad personal y familiar, se incluye en este derecho conocer, actualizar y rectificar la información recogida de las personas en bancos de datos o en archivos de entidades públicas o privadas, igualmente, en la recolección tratamiento y circulación de datos a fin de que se respeten las libertades y garantías Constitucionales de que gozan los individuos [7].

Colombia no posee una regulación normativa específica sobre ciudades inteligentes. Sin embargo, existen normas que han permitido estructurar la implementación de la tecnología a los componentes propuestos por MinTIC (competitividad, convivencia, cultura, hábitat, infraestructura, recursos y servicios). A continuación, se lleva a cabo una síntesis (ver Tabla 1) de las normas que se aplican para cada uno de los aspectos que deben ser regulados dentro de dichos componentes para la implementación de ciudades inteligentes en Colombia, a partir de la reglamentación constitucional, los cuales sirven como referente para el desarrollo jurídico de territorios inteligentes.

Tabla 1. Normas que estructuran la inmersión de ciudades inteligentes en Colombia

\section{COMPETITIVIDAD}

\begin{tabular}{|c|c|c|}
\hline $\begin{array}{l}\text { Aspecto } \\
\text { regular }\end{array}$ & Norma & Descripción \\
\hline $\begin{array}{l}\text { Gobierno } \\
\text { (en línea/ } \\
\text { abierto) }\end{array}$ & $\begin{array}{l}\text { Ley 1341, } \\
2009 \text { [8]. }\end{array}$ & $\begin{array}{l}\text { Establece principios y concep- } \\
\text { tos sobre la sociedad de la in- } \\
\text { formación y la organización de } \\
\text { las Tecnologías de la Informa- } \\
\text { ción y las Comunicaciones TIC. } \\
\text { Mediante esta norma se crea la } \\
\text { Agencia Nacional del Espectro. }\end{array}$ \\
\hline
\end{tabular}




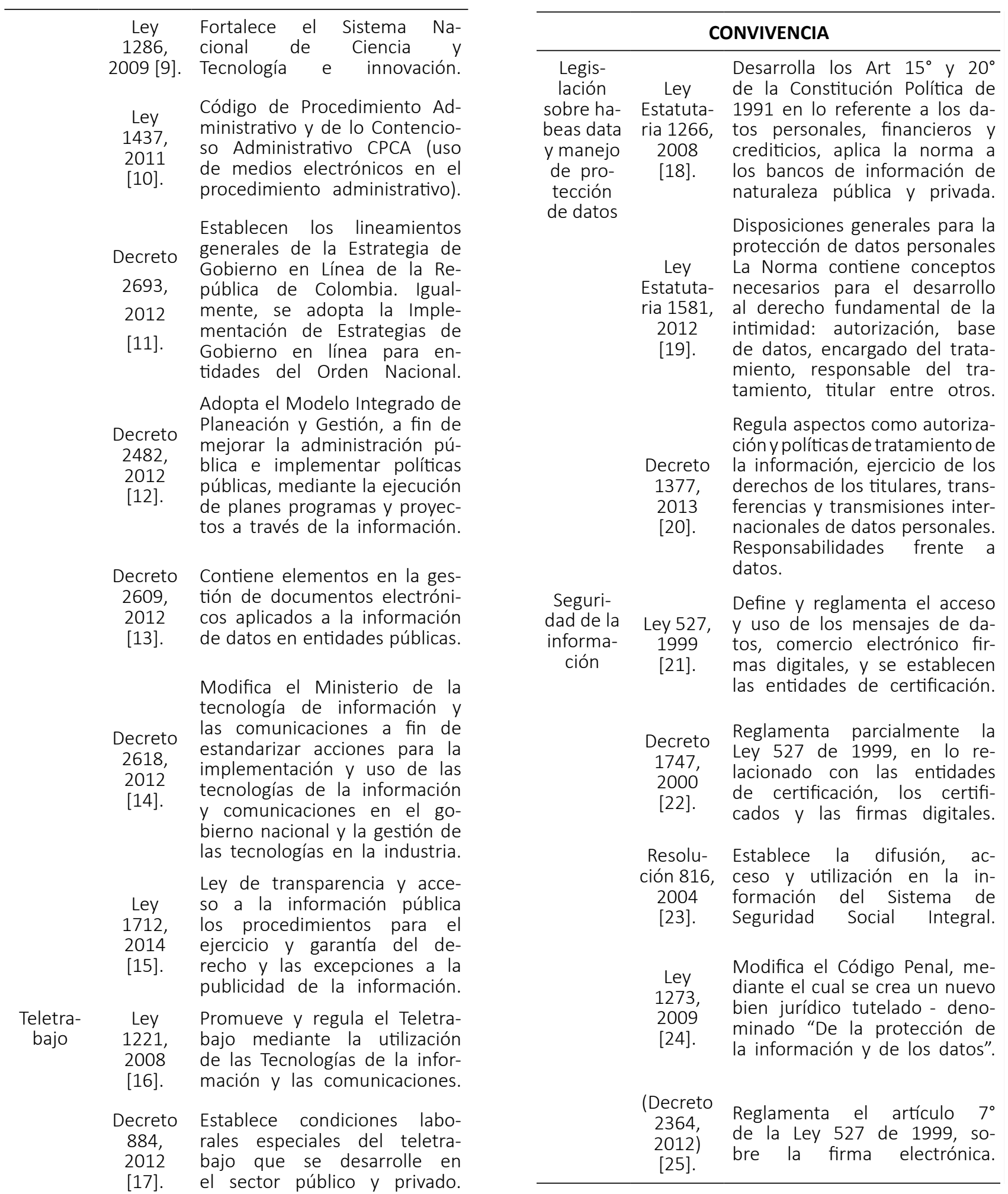




\begin{tabular}{|c|c|c|}
\hline & & HABITAT \\
\hline \multirow[t]{3}{*}{$\begin{array}{l}\text { Sosteni- } \\
\text { bilidad } \\
\text { ambien- } \\
\quad \text { tal }\end{array}$} & $\begin{array}{l}\text { Ley } 99 \\
1993 \\
{[26]}\end{array}$ & $\begin{array}{l}\text { Crea el Ministerio del Medio } \\
\text { Ambiente, se reordena el Sector } \\
\text { Público encargado de la gestión } \\
\text { y conservación del medio am- } \\
\text { biente y los recursos naturales } \\
\text { renovables, se organiza el Siste- } \\
\text { ma Nacional Ambiental - SINA. }\end{array}$ \\
\hline & $\begin{array}{l}\text { Ley } \\
1549 \\
2012 \\
{[27]}\end{array}$ & $\begin{array}{l}\text { Institucionalización de la po- } \\
\text { lítica nacional de educación } \\
\text { ambiental e incorporación } \\
\text { en el desarrollo territorial. }\end{array}$ \\
\hline & $\begin{array}{l}\text { Ley } \\
1672 \\
2013 \\
{[28]}\end{array}$ & $\begin{array}{l}\text { Regula los lineamientos para la } \\
\text { adopción de una política pública } \\
\text { de gestión integral de residuos } \\
\text { de aparatos eléctricos y electró- } \\
\text { nicos (RAEE). }\end{array}$ \\
\hline
\end{tabular}

\section{INFRAESTRUCTURA}

\begin{tabular}{|c|c|c|}
\hline \multirow[t]{3}{*}{$\begin{array}{l}\text { Desa- } \\
\text { rrollo } \\
\text { urbano y } \\
\text { territorial }\end{array}$} & $\begin{array}{l}\text { Ley } 388, \\
1997 \\
{[29] .}\end{array}$ & $\begin{array}{l}\text { Ley de Desarrollo Territorial, de- } \\
\text { fine normas de ocupación y uso } \\
\text { del suelo, que compromete los } \\
\text { intereses de todos los grupos de } \\
\text { poder presentes en el territorio. }\end{array}$ \\
\hline & $\begin{array}{l}\text { Ley } \\
1454 \\
2011 \\
{[30]}\end{array}$ & $\begin{array}{l}\text { Disposiciones sobre normas } \\
\text { orgánicas de ordenamiento te- } \\
\text { rritorial, se establece las com- } \\
\text { petencias de entidades, auto- } \\
\text { ridades, recursos, funciones y } \\
\text { servicios de los entes territoria- } \\
\text { les y su autonomía en asuntos } \\
\text { políticos, administrativos, am- } \\
\text { bientales, fiscales y geográficos }\end{array}$ \\
\hline & $\begin{array}{l}\text { Ley } \\
1469 \\
2011 \\
{[31]}\end{array}$ & $\begin{array}{l}\text { Promueve medidas para la ofer- } \\
\text { ta de suelo urbanizable y se re- } \\
\text { gulan disposiciones para acceso } \\
\text { a vivienda. }\end{array}$ \\
\hline \multicolumn{3}{|r|}{ RECURSOS } \\
\hline $\begin{array}{l}\text { Regula- } \\
\text { ción del } \\
\text { sector } \\
\text { eléctrico }\end{array}$ & $\begin{array}{l}\text { Resolu- } \\
\text { ción 038, } \\
2014 \\
\text { [32]. }\end{array}$ & $\begin{array}{l}\text { Condiciones técnicas y pro- } \\
\text { cedimientos que se aplican } \\
\text { a la medición de energía, in- } \\
\text { tercambios comerciales de } \\
\text { interconectado a nivel nacio- } \\
\text { nal e internacional y el ma- } \\
\text { nejo de protección de datos }\end{array}$ \\
\hline
\end{tabular}

\begin{tabular}{|c|c|c|}
\hline \multicolumn{3}{|r|}{ SERVICIOS } \\
\hline $\begin{array}{l}\text { Ciberse- } \\
\text { guridad }\end{array}$ & $\begin{array}{l}\text { Docu- } \\
\text { mento } \\
\text { COMPES } \\
3701 \\
2011 \\
{[33]}\end{array}$ & 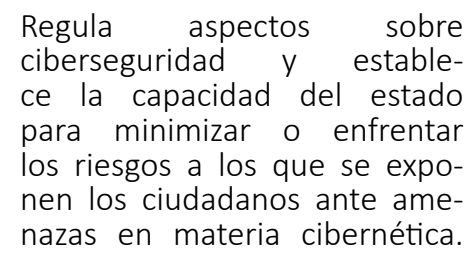 \\
\hline \multirow[t]{3}{*}{$\begin{array}{l}\text { Regula- } \\
\text { ción en } \\
\text { teleco- } \\
\text { munica- } \\
\text { ciones e } \\
\text { interope- } \\
\text { rabilidad }\end{array}$} & $\begin{array}{c}\text { CRC. Re- } \\
\text { solución } \\
3066, \\
2011 \\
{[34] .}\end{array}$ & $\begin{array}{l}\text { Incluye derechos de los usua- } \\
\text { rios de los servicios de co- } \\
\text { municaciones a fin de gozar } \\
\text { de la protección en sus datos } \\
\text { personales suministrados al } \\
\text { proveedor y que estos datos } \\
\text { no sean utilizados para fines } \\
\text { distintos de los autorizados. }\end{array}$ \\
\hline & $\begin{array}{l}\text { Resolu- } \\
\text { ción 202, } \\
2010 \\
\text { [35]. }\end{array}$ & $\begin{array}{l}\text { Define: Red de Comunica- } \\
\text { ciones, interoperabilidad e } \\
\text { interconexiones. }\end{array}$ \\
\hline & $\begin{array}{c}\text { Reso- } \\
\text { lución } \\
1301 \\
2011 \\
{[36] .}\end{array}$ & $\begin{array}{l}\text { Amplia los conceptos de ex- } \\
\text { puestos en la Resolución } 202 \text { de } \\
2010 \text {. Así mismo, indica el ob- } \\
\text { jeto de proveer redes y/o ser- } \\
\text { vicios de telecomunicaciones, } \\
\text { aplicaciones y /o contenidos. }\end{array}$ \\
\hline \multicolumn{3}{|r|}{ CULTURA } \\
\hline $\begin{array}{l}\text { Produc- } \\
\text { ción de } \\
\text { video } \\
\text { juegos, } \\
\text { libros } \\
\text { digitales } \\
\text { interac- } \\
\text { tivos y } \\
\text { series de } \\
\text { anima- } \\
\text { ción }\end{array}$ & & $\begin{array}{l}\text { No existe una norma específi- } \\
\text { ca para este componente. Sin } \\
\text { embargo, a través de los Mi- } \\
\text { nisterios de cultura y MinTIC el } \\
\text { pasado } 24 \text { de marzo se abrió } \\
\text { la convocatoria "Crea digital } \\
2017 \text { ", dirigida al sector de in- } \\
\text { dustrias culturales y creativas } \\
\text { para los mejores productos } \\
\text { en estas categorías, con fines } \\
\text { culturales y educativos [37]. }\end{array}$ \\
\hline
\end{tabular}

De igual manera, se ha expedido el Decreto 1414 de 25 de agosto de 2017, el cual modificó la estructura del MinTIC, asignando a la Dirección de Gobierno Digital funciones para formular políticas, programas y planes de adopción y apropiación de Tecnologías de la Información en las entidades del Estado [38]. En este mismo sentido, el Decreto 1008 de 14 de junio de 2018, establece los lineamientos generales de la política de Gobierno Digital [39].

El Banco Interamericano de Desarrollo (BID), toma 
como referente la ciudad de Medellín, que apunta a convertirse en ejemplo de ciudad inteligente en Colombia por su avanzada infraestructura, y tecnología, dentro de los componentes más relevantes se encuentra la simplificación de trámites, mayor y mejor movilidad, y seguridad entre otros aspectos. Igualmente, con su programa bandera "MDE: Medellín Ciudad Inteligente" busca involucrar a los ciudadanos hacia el gobierno abierto con la creación de zonas de libre acceso a Internet, a las TICs [40].

En el marco del Il seminario de Smart Cities 2017, celebrado en el mes de marzo, se incluyen ejemplos que demuestran la importancia de la implementación de las TICs en todos los ámbitos económicos, políticos, sociales y culturales de un país, muestra de ello se evidencia en México, Corea, Costa Rica, Guatemala. Brasil, Caracas, y Ecuador [41].

\section{NORMATIVA INTERNACIONAL}

La ISO ha promovido la normalización de herramientas a fin de evaluar, comparar y mejorar las prácticas a través de estándares para la implementación de las tecnologías, con el propósito de construir verdaderas ciudades inteligentes. A continuación, se describen brevemente algunas de dichas normas [40]:

\subsection{ISO 37101}

"Sustainable development and resilience of communities - Management systems - General principles and requirements". Sistemas de gestión para el desarrollo de comunidades sostenibles y resilientes.

\subsection{ISO 37120}

"Sustainable development and resilience of communities - Global city indicators for city services and quality of life". Indicadores de servicios para la ciudad relacionadas con servicios de la ciudad y calidad de vida.

En Colombia ICONTEC es reconocido por el gobierno nacional como organismo de normalización mediante el Decreto 2263 de 1993, y en materia de seguridad de la información se aprueban las normas técnicas tales como: NTC-ISO-IEC 27001 por medio del cual se establecen requisitos para la implementación, mantenimiento y mejora permanente en la gestión de la seguridad de la información; NTC-ISO-IEC 27002 que establece directrices y principios de seguridad de la información organizacional, teniendo en cuenta los entornos del riesgo de seguridad de la información en una organización [41].

\section{CONCLUSIONES}

El Gobierno Nacional a través del MinTIC, se ha propuesto desarrollar políticas públicas que permitan fomentar el desarrollo de ciudades inteligentes en sus siete componentes (competitividad, convivencia, cultura, hábitat, infraestructura, recursos y servicios). Se ha llevado a cabo una revisión de literatura presentando una síntesis de las normas, leyes, decretos, y resoluciones entre otras, que sirven como referente para la aplicación de dichos componentes, los cuales han permitido la puesta en marcha de este proyecto gubernamental, a fin de mejorar la calidad de vida de los ciudadanos a través del uso de las tecnologías y las comunicaciones.

\section{REFERENCIAS}

[1] Unión Internacional de Telecomunicaciones, (2016). Construir las ciudades inteligentes y sostenibles del mañana. Actualidades de la UIT, 2, pp. 1-29.

[2] MinTIC, 2016. [Online]. Available: http://www. mintic.gov.co/portal/604/w3-article-15448.html

[3] MinTIC, $2017 . \quad$ [Online]. Available: http://estrategiaticolombia.co/ciudadesinteligentes/\#competitividad---convivencia

[4] ISO 37120, 2014. [Online]. Available: https://www.iso.org/standard/62436.htm I

[5] CEN-CENELEC, 2017. [Online]. Available: https://www.cencenelec.eu/standards/Sectors/ SmartLiving/smartcities/Pages/SSCC-CG.aspx

[6] AENOR, 2015. [Online]. Available: http://www. agendadigital.gob.es/planes-actuaciones/Bibliotecaciudadesinteligentes/Material\%20complementario/normas_ciudades_inteligentes.pdf 
[7] Constitución Política de Colombia, 1991. [Online]. Available:http://www.corteconstitucional.gov.co/ inicio/Constitucion\%20politica\%20de\%20Colombia\%20-\%202015.pdf

[8] Ley 1341, 2009. [Online]. Available:http://www. mintic.gov.co/portal/604/articles-3707_documento.pdf

[9] Ley 1286, 2009. [Online]. Available: http://www.colciencias.gov.co/sites/default/files/ upload/reglamentacion/ley-1286-2009.pdf

[10] Ley 1437, 2011. [Online]. Available: http://www.alcaldiabogota.gov.co/sisjur/normas/ Norma1.jsp?i=41249

[11] Decreto 2693, 2012. [Online]. Available: http://www.mintic.gov.co/portal/604/w3-article-3586.html

[12] Decreto 2482, 2012. [Online]. Available: http://www.mintic.gov.co/portal/604/articles-3581_documento.pdf

[13] Decreto 2609, 2012. [Online]. Available: https://www.mintic.gov.co/portal/604/articles-3528_documento.pdf

[14] Decreto 2618, 2012. [Online]. Available: http://www.mintic.gov.co/portal/604/articles-3529_documento.pdf

[15] Decreto 2618, 2012. [Online]. Available: http://www.mintic.gov.co/portal/604/articles-3529_documento.pdf

[16] Ley 1221, 2008. [Online]. Available: https://www.mintic.gov.co/portal/604/articles-3703_documento.pdf

[17] Decreto 884, 2012. [Online]. Available: http://www.mintic.gov.co/portal/604/articles-3638 documento.pdf

[18] Ley Estatutaria 1266. 2008. [Online]. Available: http://www.alcaldiabogota.gov.co/sisjur/normas/ Norma1.jsp?i=43747

[19] Ley Estatutaria 1581, 2012. [Online]. Available: http://wsp.presidencia.gov.co/Normativa/Leyes/ Documents/LEY\%201581\%20DEL\%2017\%20DE\%20 OCTUBRE\%20DE\%202012.pdf

[20] Decreto 1377, 2013. [Online]. Available: https://www.mintic.gov.co/portal/604/articles-4274_documento.pdf

[21] Ley 527, 1999. [Online]. Available: http://www.mintic.gov.co/portal/604/w3-article-3679.html

[22] Decreto 1747, 2000. [Online]. Available: http:// www.alcaldiabogota.gov.co/sisjur/normas/Norma1. jsp?i=4277

[23] Ministerios de Salud. Resolución 816, 2004. [Online]. Available: https://www.notinet.com.co/pedidos/816min.pdf

[24] Ley 1273, 2009. [Online]. Available: http://www. mintic.gov.co/portal/604/w3-article-3705.html

[25] Decreto 2364, 2012. [Online]. Available: http:// www.alcaldiabogota.gov.co/sisjur/normas/Norma1. jsp?i=50583

[26] Ley 99, 1993. [Online]. Available: http://www.bogotajuridica.gov.co/sisjur/normas/Norma1.jsp?i=297

[27] Ley 1549, 2012. [Online]. Available: http://www. alcaldiabogota.gov.co/sisjur/normas/Norma1.jsp? $\mathrm{i}=48262$

[28] Ley 1672, 2013. [Online]. Available: http://www. andi.com.co/RelNor/Documents/Ley\%201672\%20 de\%202013.pdf

[29] Ley 388, 1997. [Online]. Available: http://www.alcaldiabogota.gov.co/sisjur/normas/Norma1.jsp?i=339

[30] Ley 1454, 2011. [Online]. Available: http://www.senado.gov.co/images/stories/Dependencias/Comision_ordenamiento/LEY_1454_DE_ORDENAMIENTO_TERRITORIAL.pdf

[31] Ley 1469, 2011. [Online]. Available: http://wsp. presidencia.gov.co/Normativa/Leyes/Documents/ ley146930062011.pdf

[32] Comisión de regulación de Energía y Gas (GREG). Resolución 038, 2014. [Online]. Available: http:// apolo.creg.gov.co/Publicac.nsf/1c09d18d2d5ffb5b05256eee00709c02/0131f0642192a5a205257cd800728c5e/\$FILE/Creg038-2014.pdf

[33] Documento COMPES 3701, 2011. [Online]. Available: http://www.mintic.gov.co/portal/604/articles-3510_documento.pdf CRC.

[34] Resolución 3066, 2011. [Online]. Available: http://www.alcaldiabogota.gov.co/sisjur/normas/ 
Norma1.jsp?i $=42871$

[35] MinTIC. Resolución 202, 2010. [Online]. Available: http://www.mintic.gov.co/portal/604/w3-article-3762.html

[36] CRC. Resolución 1301, 2011. [Online]. Available: http://www.alcaldiabogota.gov.co/sisjur/normas/ Norma1.jsp?i $=43747$

[37] Ministerio de Cultura, 2017. [Online]. Available: http://www.mincultura.gov.co/prensa/noticias/ Paginas/MinCultura-y-MinTIC-abren-la-convocatoria-Crea-Digital-que-entregar\%C3\%A1-\$2.000-millones-para-proyectos-de-Contenidos-Digitales.aspx

[38] Decreto 1414, 2017. [Online]. Available: https:// www.mintic.gov.co/portal/604/w3-article-57805. html

[39] Decreto 1008, 2018. [Online]. Available: https:// www.mintic.gov.co/portal/604/w3-article-74903. html

[40] D. Amar Florez, "Estudios de casos internacionales de ciudades inteligentes: Medellín, Colombia", 2016. DOI. http://dx.doi.org/10.18235/0000406

[41] II seminario de Smart Cities, 2017. [Online]. Available: https://colaboracion.dnp.gov.co/CDT/ Vivienda\%20Agua\%20y\%20Desarrollo\%20Urbano/ Presentaciones/Seminario\%20Smart\%20Cities/11_ JOSE\%2OMORALES_Post\%2OHIII.pdf?

[42] MinTIC, 2014. [Online]. Available: http://centrodeinnovacion.mintic.gov.co/es/investigaciones/politica-tic-para-ciudades-yoterritorios-inteligentes

[43] UPME, 2016. [Online]. Available: http://www1. upme.gov.co/Paginas/Smart-Grids-Colombia-Visi\%C3\%B3n-2030.aspx 